\title{
Iron Chelation as a Possible Mechanism for Aspirin-induced Malondialdehyde Production by Mouse Liver Microsomes and Mitochondria
}

\author{
K. B. Schwarz, B. J. Arey, K. Tolman, and S. Mahanty \\ Department of Pediatrics, Division of Pediatric Gastroenterology, St. Louis University School of Medicine, Cardinal Glennon Children's \\ Hospital Pediatric Research Institute, St. Louis, Missouri 63104
}

\begin{abstract}
To investigate the possibility that lipid peroxidation is the mechanism responsible for aspirin-induced liver damage, pure neutralized acetylsalicylic acid (ASA), 0.6-90.9 $\mathrm{mM}$, was added to calcium-aggregated mouse liver microsomes followed by incubation in NADPH buffer at $37^{\circ} \mathrm{C}$ for 60 min and subsequent measurement of malondialdehyde (MDA). MDA production at ASA concentrations from 1.2 to $4.6 \mathrm{mM}$ was greater than control $(P<0.004)$. Peak MDA values were observed with $4.6 \mathrm{mM}$ ASA, $39.58 \pm 6.73 \mathrm{nmol} \mathrm{MDA} / \mathrm{mg}$ protein vs. $16.16 \pm 2.85(P<0.004)$. Higher concentrations of ASA were inhibitory compared with the value at $4.6 \mathrm{mM}(P<0.001)$. Aspirin had similar effects on MDA production by mouse liver mitochondria. MDA production with either ASA or buffer was completely suppressed by the potent iron-chelating agents desferrioxamine and alpha,alpha' dipyridyl when these were added to the microsomal preparations. Since MDA production in this system is known to be affected by iron-chelating agents (enhanced at low concentration, inhibited at higher concentration), the iron-chelating properties of ASA were investigated. Conductivity titration curves of $\mathrm{Fe}(\mathrm{OH})_{3}$ added to water or ASA suggested that the ASA was complexing with iron. The presence of an iron-ASA complex was established by high pressure liquid chromatographic analysis of the solution from this study. We conclude that aspirin enhances MDA production by hepatic microsomes and mitochondria via an aspiriniron chelate and that this represents at least one mechanism by which aspirin may produce liver damage.
\end{abstract}

\section{Introduction}

Although the hepatatoxic potential of aspirin was first described in 1971 (and over 300 cases of aspirin-related hepatic injury have been reported), the precise mechanism of injury remains unknown (4). However, some evidence has accumulated that salicylates have an injurious effect on hepatocyte plasma and organelle membranes, resulting in the loss of important metabolites from the cell. For example, Kaplowitz et

Portions of this work have been presented to the American Association for the Study of Liver Diseases (1), the National Reye's Syndrome Foundation (2), and the American Pediatric Society and the Society for Pediatric Research (3).

Address reprint requests and all correspondence to Dr. K. B. Schwarz, Johns Hopkins University School of Medicine, Brady 303, 600 N. Wolfe St., Baltimore, MD 21205.

Received for publication 9 March 1987 and in revised form 12 August 1987.

J. Clin. Invest.

(c) The American Society for Clinical Investigation, Inc.

$0021-9738 / 88 / 01 / 0165 / 06 \$ 2.00$

Volume 81 , January $1988,165-170$ al. (5) have observed decreased hepatic glutathione in rats given aspirin or sodium salicylate by the intraperitoneal route. These workers demonstrated that incubation of liver slices in the presence of sodium salicylate $(100 \mathrm{mg} / \mathrm{dl})$ was associated with leakage of glutathione from liver into the supernate. Aspirin has no effect on the enzymes regulating the synthesis and catabolism of glutathione. Similarly, Tolman et al. (6) reported that administration of graded doses of sodium salicylate to rat liver cells cultured in monolayers was associated with loss of lactic dehydrogenase into the culture media. Finally, Martens and Lee (7) demonstrated that incubation of isolated hepatic mitochondria with salicylate plus calcium induced loss of $90 \%$ of the intramitochondrial $\mathrm{NAD}^{+}$and $\mathrm{NADP}^{+}$into the surrounding medium.

Peroxidation of membrane long chain polyunsaturated fatty acids is a common mechanism whereby membrane damage can occur (8). Since aspirin can stimulate lipid peroxidation in erythrocytes incubated in the presence of an oxidant stress $\left(\mathrm{H}_{2} \mathrm{O}_{2}\right)(9)$, the possibility that aspirin might also stimulate lipid peroxidation in hepatic microsomes and mitochondria was investigated.

Hunter et al. (10) have demonstrated that hepatic mitochondria form lipid peroxides when exposed to even minute amounts of iron. The central role of iron in stimulating NADPH-dependent lipid peroxidation in hepatic microsomes was first described in 1964 by Hochstein et al. (11). Subsequently Wills (12) observed that no other metal ion could adequately replace inorganic iron in this system. Utilizing a model system made from extracted microsomal lipids as substrates for peroxidation, Tien et al. (13) noted that addition of the iron-chelating agent EDTA to this system enhanced lipid peroxidation. Thus, in the present study, after we had described the ability of aspirin to enhance hepatic mitochondrial and microsomal lipid peroxidation, the possibility that aspirin could chelate iron was investigated.

\section{Methods}

\section{Microsomal malondialdehyde $(M D A)^{1}$ production}

Livers were obtained from male, Balb $\mathrm{C}$ mice nourished with mouse chow (Ralston-Purina Co., St. Louis, MO) and given ad libitum water. Average mouse weight was $18.4 \pm 0.2 \mathrm{~g}$. Food and water were withdrawn just before killing by cervical dislocation and rapid hepatectomy was performed. Calcium aggregated microsomes were obtained by a modification of the method of Roders et al. (14) as follows: a $10 \%$ wt/vol whole liver homogenate in $0.154 \mathrm{M} \mathrm{NaCl}$ was centrifuged at 2,700 $\mathrm{g}$ for $10 \mathrm{~min}$ (Accuspin FR centrifuge with a TH-4 horizontal rotor with buckets; Beckman Instruments, Inc., Fullerton, CA). (At this high ionic strength, the mitochondria are completely sedimented. ${ }^{2}$

1. Abbreviations used in this paper: ASA, acetylsalicylic acid; MDA, malondialdehyde.

2. Recknagel, R. O., personal communication. 
The postmitochondrial supernatant fraction was diluted 1:2 (vol/vol) with $0.154 \mathrm{M} \mathrm{NaCl}$, and $1.0 \mathrm{M} \mathrm{CaCl}_{2}$ was added for a final concentration of $10 \mathrm{mM} \mathrm{Ca}^{++}$. After thorough mixing, the suspension was centrifuged at $20,000 \mathrm{~g}$ for $15 \mathrm{~min}$ (J2-21 centrifuge with JA-17 rotor; Beckman Instruments, Inc.). The resulting pellet was resuspended by sonication and diluted (10\% wt/vol) with phosphate-buffered saline (0.51 mM KH $2 \mathrm{PO}_{4}, 3.44 \mathrm{mM} \mathrm{K}_{2} \mathrm{HPO}_{4}$, and $150 \mathrm{mM} \mathrm{NaCl}, \mathrm{pH} 6.6$ ). This yielded a suspension containing $100 \mathrm{mgeq}$ calcium aggregated microsomes per milliliter. (A milligram equivalent of microsomes is that amount of microsomes derived from $1 \mathrm{mg}$ wet weight of whole liver.) All work involved with isolation of the microsomes was carried out at $4^{\circ} \mathrm{C}$.

Microsomes were incubated aerobically in a water bath with agitation in a system containing $0.3 \mathrm{mM}$ NADPH, phosphate-buffered saline (as above), and $25 \mathrm{mgeq}$ calcium aggregated microsomes per milliliter, at $37^{\circ} \mathrm{C}$ (values refer to final concentration). Just before incubation, neutralized (pH 7.0) acetylsalicylic acid (ASA) (2-acetoxybenzoic acid) (Sigma Chemical Co., St. Louis, MO) in phosphate-buffered saline or an equal volume of buffer alone was added to the microsomal incubation media. According to the manufacturer, ASA was of the highest $(>99 \%)$ purity available. MDA was measured after 60 min of incubation by the thiobarbituric acid method (15). Results were expressed on the basis of microsomal protein analyzed (16) before the addition of aspirin to the incubate. The standard curve for MDA was measured by thiobarbituric acid reacting with an acidic ethanolic solution of tetraethoxypropane. To determine if aspirin affected either this reaction or the absorbance value of the product, the MDA standard curve was conducted in the presence of aspirin in concentrations of $0.16,0.32,1.3,2.5$, and $17.6 \mathrm{mM}$. Aspirin in these concentrations had no effect on the MDA standard curve. To test the possibility that obtaining the microsomes via calcium aggregation affected the results, similar experiments were performed with microsomes obtained by the conventional method of sequential differential centrifugation (17). MDA production in microsomes obtained in this fashion and incubated with $2.3 \mathrm{mM}$ ASA was $44.6 \pm 1.7 \mathrm{nmol} \mathrm{MDA}(n=6)$ vs. $15.9 \pm 1.1$ $(n=6)$ for controls $(P<0.001)$ (similar to values described in the Results section for calcium-aggregated microsomes).

In some experiments with calcium-aggregated microsomes, an NADPH-generating system (18) was used in place of NADPH, utilizing $5 \mathrm{mM} \mathrm{MgCl}, 3 \mathrm{mM}$, L-isocitrate, $0.07 \mathrm{U}$ isocitrate dehydrogenase $/ \mathrm{ml}, 100 \mathrm{mM}$ NADP, and $2.5 \mathrm{mM}$ nicotinamide (quantities expressed as final concentrations) in the incubation mixture. When the generating system was used with calcium-aggregated microsomes, results were similar to those obtained with NADPH: $39.03 \pm 0.96 \mathrm{nmol}$ $\mathrm{MDA} / \mathrm{mg}$ protein in microsomes incubated with $2.3 \mathrm{mM}$ ASA $(n=6)$ vs. $16.37 \pm 0.43$ for controls $(n=6) P<0.001$.

\section{Mitochondrial MDA production}

Livers were obtained from male Balb $\mathrm{C}$ mice as described for the microsomal studies. Mitochondria were isolated according to a modification of the method by Hogeboom (19). A $10 \% \mathrm{wt} / \mathrm{vol}$ whole liver suspension was homogenized in $0.25 \mathrm{M}$ sucrose, $\mathrm{pH} 7.4$, using a loose-fitting pestle in a $15-\mathrm{ml}$ Wheaton glass tissue grinder. The homogenate was layered carefully over an equal volume of $0.34 \mathrm{M} \mathrm{su}-$ crose and centrifuged (model J2-2 centrifuge with a JA 17 rotor; Beckman Instruments, Inc.) at $700 \mathrm{~g}(1,800 \mathrm{rpm})$ for $10 \mathrm{~min}$. The supernatant fraction was centrifuged at $5,000 \mathrm{~g}(6,100 \mathrm{rpm})$ for $10 \mathrm{~min}$. The mitochondrial pellet was resuspended (5\% wt/vol) in $0.25 \mathrm{M}$ sucrose and centrifuged twice at $24,000 \mathrm{~g}(13,250 \mathrm{rpm})$ for $10 \mathrm{~min}$, utilizing fresh $0.25 \mathrm{M}$ sucrose between centrifugations. The supernatant fraction was discarded and the resulting pellet was resuspended and diluted ( $5 \% \mathrm{wt} / \mathrm{vol})$ with phosphate-buffered saline as described for the microsomes. From then on, the procedure was identical to that described for the latter organelles, with the exception that the concentration of NADPH was $0.6 \mathrm{mM}$. The incubate contained $\sim 2-3 \mathrm{mg}$ mitochondrial protein per milliliter. The iron content of all solutions was determined by the ferrozine method $(20,21)^{2}$ (see below). Any solutions containing measurable iron were passed over an iron-chelating resin
(Chelex 100; Bio-Rad Laboratories, Richmond, CA) and the absence of iron verified by the ferrozine method $(20,21){ }^{2}$ All work involved with isolation of the mitochondria was carried out at $4^{\circ} \mathrm{C}$.

\section{Ferrozine procedure for determination of iron}

The stock solution of aspirin was analyzed for the presence of ferric (Fe III) and ferrous (Fe II) iron by the method of Stookey (20) and Carter (21) as modified by Recknagel. ${ }^{2}$ An Fe III standard was used to verify the efficiency of the reducing agent (hydroxylamine) and an Fe II standard was used to assure that the ferrous iron complex with ferrozine would be detected spectrophotometrically. (Ferrozine is the salt of 3-(2-pyridyl)-5,6-bis(4-phenylsulfonic acid)-1,2,4-triazine).

\section{Conductimetric titration}

Titration was performed by the dropwise addition of a $\mathrm{Fe}(\mathrm{OH})_{3}$ solution to deionized water or to a solution of $3 \%$ ASA. Conductivity was measured with a conductivity meter (4503; Markson Science Inc., Phoenix, AZ).

\section{Synthesis of iron-aspirin chelate}

The method of Williams et al. (22) was modified with substitution of ferrous sulfate for copper sulfate pentahydrate. ASA ( $3.60 \mathrm{~g}, 0.02 \mathrm{~mol}$ ) was allowed to dissolve in a solution of potassium bicarbonate $(2.02 \mathrm{~g}$, $0.02 \mathrm{~mol}$ ) in $30 \mathrm{ml}$ water. To this solution was added $\mathrm{FeSO}_{4} \cdot 7 \mathrm{H}_{2} \mathrm{O}$ $(2.78 \mathrm{~g}, 0.01 \mathrm{~mol})$ in $20 \mathrm{ml}$ water, slowly and with constant stirring. The aquamarine precipitate that formed was filtered under an $\mathbf{N}_{2}$ atmosphere and vacuum dried overnight at room temperature in a dessicator containing calcium chloride. Subsequently, the dried precip-

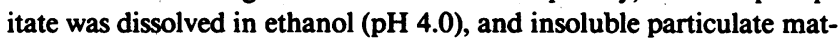
ter was removed using a Sep-pak $\mathrm{C}_{18}$ unit (Waters) by elution with $\mathrm{H}_{2} \mathrm{O} / \mathrm{MeOH} / \mathrm{CH}_{3} \mathrm{COOH}$ 50:48:1 (vol/vol/vol).

\section{High pressure liquid chromatographic identification of aspirin and aspirin-iron chelate (23)}

Pure neutralized ASA was dried under $\mathrm{N}_{\mathbf{2}}$, dissolved in methanol, and injected directly onto column. The solution (of $\mathrm{Fe}(\mathrm{OH})_{3}$ and ASA) from the conductimetric titration study was prepared for HPLC by passage through a Sep-pak $C_{18}$ unit (see above) to remove particulate matter. HPLC was carried out using a pump (model 501) attached to an ultraviolet-Vis LC spectrophotometer (model 481) and data module (model 740; Millipore, Waters Chromatography Division, Milford, MA). Samples of ASA alone, the conductimetric titration solution, and the iron-ASA chelate, were injected onto a Nova-pac $C_{18}$ column via an injector (model UK6; Millipore, Waters Chromatography Div.)

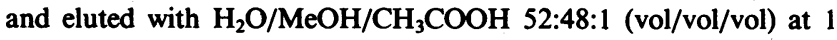
$\mathrm{ml} / \mathrm{min}$.

Results were calculated as $k^{\prime}=\left(V_{s}-V_{0}\right) / V_{0}$, where $V_{s}$ is volume of peak in question and $V_{0}$, void column.

\section{Statistics}

Data are given as mean \pm SE. Nonpaired two-tailed student's $t$ test was used to compare treatment vs. control. One-way analysis of variance was used to compare results using various concentrations of ASA. Significance level was $P<0.05$.

\section{Results}

The results of addition of varying concentrations of ASA on MDA synthesis by microsomes are shown in Fig. 1. The data demonstrate an increase in MDA production as medium ASA concentration was increased up to $4.6 \mathrm{mM}$. Higher concentrations of ASA were inhibitory compared with the value at 4.6 $\mathrm{mM}(P<0.001)$. Time course data in Fig. 2 demonstrate that the effect of ASA on MDA production was already apparent by $15 \mathrm{~min}$. When microsomes were incubated with salicylic acid (a major metabolite of aspirin) (24) in place of aspirin, increased MDA production was observed. $24.51 \pm 5.53 \mathrm{nmol}$ 


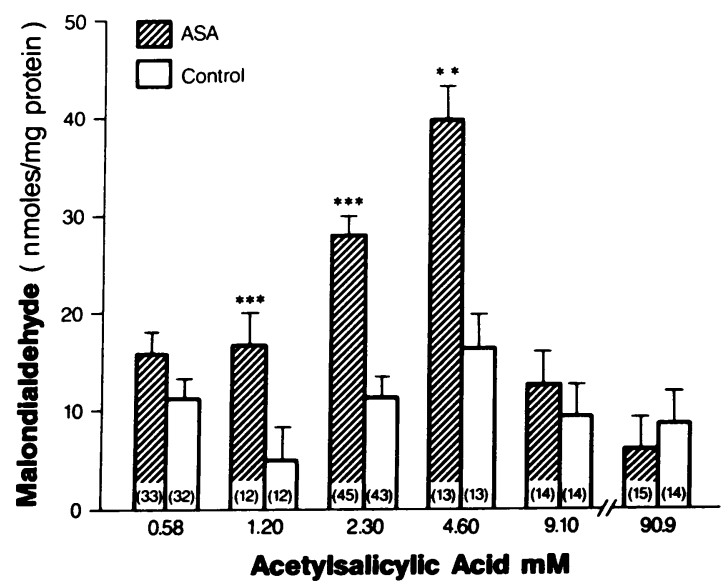

Figure 1. The effect of various concentrations of pure neutralized ASA on MDA production was studied in calcium-aggregated mouse liver microsomes (14) incubated in the presence of $0.3 \mathrm{mM} \mathrm{NADPH}$ at $37^{\circ} \mathrm{C}$ for $60 \mathrm{~min}$. The concentrations of aspirin (expressed in millimolar above) would correspond to the following values: 10, 22, 42, 83,164 , and $1,636 \mathrm{mg} / \mathrm{dl}$. Symbols for statistical significance utilized in Figs. 1,3 , and 4 are as follows: ${ }^{*} P<0.05,{ }^{* *} P<0.01$, and ${ }^{* * *} P$ $<0.001$ for treated vs. control. $n$, Nos. in parentheses.

$\mathrm{MDA} / \mathrm{mg}$ protein for $2.3 \mathrm{mM}$ salicylic acid $(n=9)$ compared with $9.66 \pm 1.12$ for controls $(P<0.02)(n=9)$. However, when a minor metabolite of aspirin, gentisic acid, (24) was used in place of aspirin, there was inhibition of MDA formation. Results were $7.28 \pm 1.28 \mathrm{nmol} \mathrm{MDA} / \mathrm{mg}$ protein for microsomes incubated with $2.3 \mathrm{mM}$ gentisic acid $(n=14)$ compared with $12.30 \pm 1.01(P<0.004)(n=15)$ for controls.

Since iron contamination of buffers in microsomal preparations is common (25), and would promote lipid peroxidation, the possibility that the stock solution of ASA was contaminated with iron was investigated. The stock solution of aspirin contained $0.2 \mu \mathrm{g}$ iron $/ \mathrm{ml}$. Thus, the final iron concentration in the microsomal incubation medium was only 0.1 $\mu \mathrm{M}$ when an ASA stock solution was used to achieve a final incubate ASA concentration of $2.3 \mathrm{mM}$. When microsomes were incubated with this amount of ASA, results were $27.16 \pm 1.82 \mathrm{nmol} \mathrm{MDA} / \mathrm{mg}$ protein vs. $3.36 \pm 0.99$ with 0.1 $\mu \mathrm{M} \mathrm{FeSO}_{4}(P<0.001)$. To ascertain that the trace iron contaminant in the ASA solution was not acting synergistically with ASA to enhance MDA production, all iron was removed from ASA stock solution (as well as all buffers and reagents used) by passage of these solutions over Chelex 100. Absence of iron in the resin treated solutions was verified with the

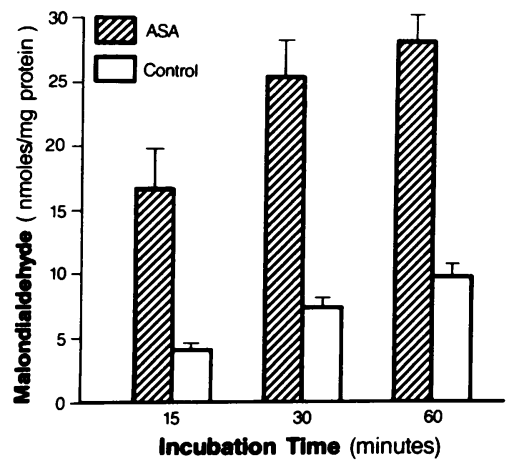

Figure 2. A time course study of MDA production by calcium-aggregated hepatic microsomes (see Fig. 1) treated with $2.3 \mathrm{mM}$ ASA or buffer. ferrozine method $(20,21)$. When the treated solutions were used, results for $2.3 \mathrm{mM}$ ASA were $22.05 \pm 0.61 \mathrm{nmol} \mathrm{MDA} /$ $\mathrm{mg}$ protein for ASA $(n=6)$ vs. $8.41 \pm 0.49$ for controls $(n=6)$ $(P<0.001)$.

The effect of desferrioxamine (a potent iron-chelating agent and superoxide scavenger) (26) on MDA production by microsomes was studied. As shown in Fig. 3, when microsomes were incubated with buffer and desferrioxamine or aspirin plus desferrioxamine, MDA production was almost completely abolished. Similar results were obtained when alpha',alpha dipyridyl (an iron chelator without superoxide scavenging activity) was used in place of desferrioxamine.

Data in Fig. 4 indicate that aspirin also affected MDA production by mouse liver mitochondria studied over a similar range of aspirin concentrations as those employed in the microsomal studies. In the mitochondria as well as in microsomes, aspirin at low concentration stimulated MDA production while it inhibited the process at the higher concentration.

To study the possibility that ASA may be forming a complex with iron to bring about the observed increase in MDA production, the effect of ASA on the electrical conductance of $\mathrm{Fe}(\mathrm{OH})_{3}$ was analyzed. In Fig. 5, it can be seen that addition of ferric hydroxide to deionized water resulted in a linear increase in conductance directly proportional to the concentration of $\mathrm{Fe}(\mathrm{OH})_{3}$. When the same conductimetric titration was performed with $\mathrm{Fe}(\mathrm{OH})_{3}$ added to a $3 \%(0.166 \mathrm{M})$ ASA solution, there was no increase in conductance until the molarity of the $\mathrm{Fe}(\mathrm{OH})_{3}$ exceeded that of the ASA solution.

When an aliquot from the conductimetric titration study described above was injected onto a reverse phase HPLC column (see Fig. 6), there appeared two species from the $\mathrm{Fe}(\mathrm{OH})_{3}$-aspirin solution, the first peak with $k^{\prime}=1.10$, and a second with $k^{\prime}=2.00$. When the stock solution of aspirin was studied in similar fashion by HPLC, a single peak with $k^{\prime}$ $=1.08$ was observed. Fig. 6 also illustrates an HPLC tracing from an experiment in which the method of Williams et al. (22) was utilized to produce an iron chelate complex as a precipitate. The precipitate was subsequently resuspended in

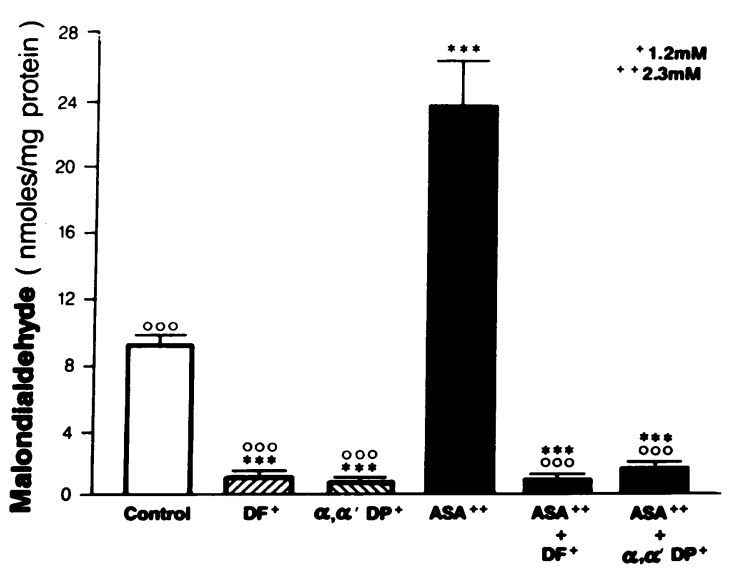

Figure 3. The effect of the iron-chelating agents desferrioximine (DF) and alpha,alpha' dipyridyl ( $\alpha, \alpha^{\prime}$ DP) on MDA production by calcium-aggregated mouse liver microsomes (14) was studied in the absence (control) or presence of $2.3 \mathrm{mM}$ ASA added to the microsomal suspension just before incubation in the presence of $0.3 \mathrm{mM}$ $\mathrm{NADPH}$ at $37^{\circ} \mathrm{C}$ for $60 \mathrm{~min}$. Open circles compare results to ASA alone and asterisks are used for comparison with control. 




Figure 4. The effect of various concentrations of pure neutralized ASA on MDA production was studied in mouse liver mitochondria incubated in the presence of $0.6 \mathrm{mM} \mathrm{NADPH}$ at $37^{\circ} \mathrm{C}$ for $60 \mathrm{~min}$.

eluting solvent and injected onto the same column. There again appeared two species with $k^{\prime}$ value of the first very similar to that of aspirin alone; $k^{\prime}$ of the second peak suggested the presence of a complex different than that obtained from the conductimetric titration experiment.

\section{Discussion}

The results of our studies demonstrate that addition of pure neutralized ASA to mouse liver microsomes or mitochondria incubated in the presence of NADPH is associated with a roughly dose-dependent increase in MDA production over the range of concentrations from 1.2 to $4.6 \mathrm{mM}$ (equivalent to $22-83 \mathrm{mg} / \mathrm{dl}$ ). These results are consistent with observations by Stockman et al. (9) of increased MDA production by erythrocytes incubated in the presence of aspirin and hydrogen peroxide. Regillo et al. (27) also postulated a possible role for lipid peroxidation in aspirin-induced gastric injury.

Our studies have provided three separate lines of evidence that aspirin can complex with iron: the conductimetric titration study, the HPLC analyses, and the effect of iron chelators on ASA-induced hepatic microsomal MDA production. Martell et al. (28) have commented on the utility of electrical conductance measurements for studying the properties of chelates; our conductimetric titration study clearly demonstrates that aspirin can complex with iron. The HPLC studies of both the titration solution and the synthetic iron-aspirin chelate provide further evidence of such a complex. The results of our

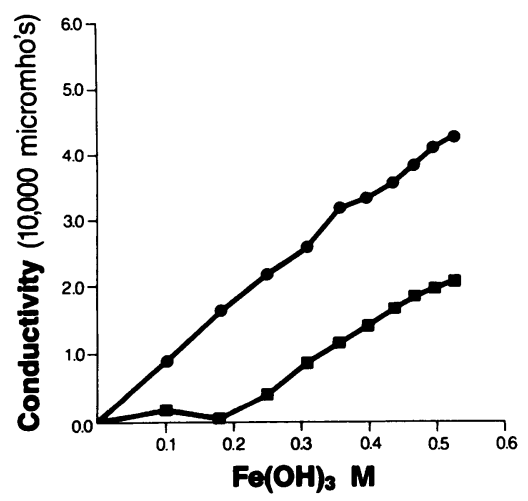

Figure 5. Effect of ASA on conductivity of a solution of $\mathrm{Fe}(\mathrm{OH})_{3}$. Conductimetric titration was performed with a conductivity meter (Markson Science Inc.) with dropwise addition of a solution of $\mathrm{Fe}(\mathrm{OH})_{3}$ to deionized $\mathrm{H}_{2} \mathrm{O}(-\bullet-)$ or to a solution of $3 \%$ ASA (一- -).

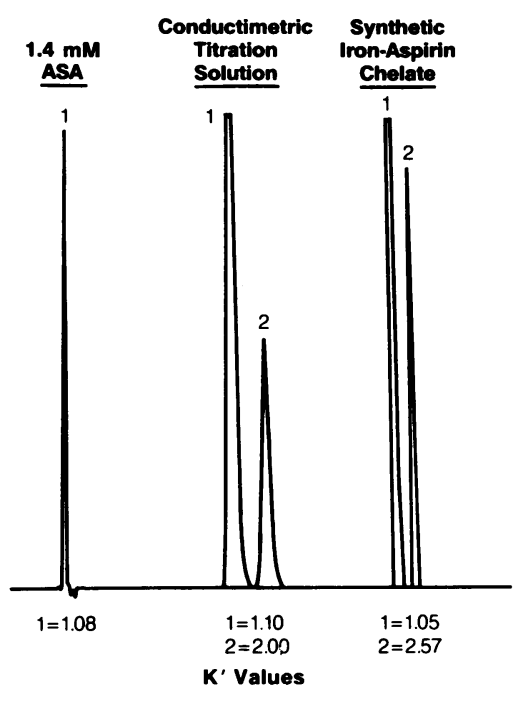

Figure 6. HPLC chromatograms of the 1.4 $\mathrm{mM}$ pure neutralized ASA analyzed by the method of Ali (23), the conductimetric titration solution shown in Fig. 5 containing $0.6 \mathrm{M}$ $\mathrm{Fe}(\mathrm{OH})_{3}$ in $0.17 \mathrm{M}$ ASA, and the putative iron-aspirin chelate synthesized by the method of Williams et al. (22). Samples were eluted with $\mathrm{H}_{2} \mathrm{O}$ / $\mathrm{MeOH} / \mathrm{CH}_{3} \mathrm{COOH}$, $52: 48: 1(\mathrm{vol} / \mathrm{vol} / \mathrm{vol})$ at $1 \mathrm{ml} / \mathrm{min}$.

studies with desferrioxamine and alpha,alpha' dipyridyl also suggest that aspirin-induced lipid peroxidation is mediated via iron.

Tien et al. (13) have reviewed the various ways in which the chelating agent EDTA can influence lipid peroxidation. When the molar ratio of EDTA-iron was less than one, the agent enhanced lipid peroxidation in phospholipid liposomes. In systems utilizing $\mathrm{Fe}^{2+}$, it was thought that EDTA chelation of $\mathrm{Fe}^{2+}$ facilitated the autoxidation of $\mathrm{Fe}^{2+}$ leading to lipid peroxidation via reduction of hydrogen peroxide to form the hydroxyl radical that initiated the peroxidation. In systems utilizing the $\mathrm{Fe}^{3+}$, the chelating agent was thought to enhance lipid peroxidation via facilitation of the reduction of $\mathrm{Fe}^{3+}$. When the concentration of EDTA exceeded that of $\mathrm{Fe}^{3+}$ in a xanthine oxidase reaction mixture containing ADP- $\mathrm{Fe}^{3+}$, lipid peroxidation was inhibited, probably as a consequence of the EDTA removing the $\mathrm{Fe}^{3+}$ from the ADP. Our results of stimulation of MDA production by aspirin in liver microsomes or mitochondria at relatively low ASA concentrations and inhibition of MDA production at high ASA concentrations are similar to the concentration dependent effects of EDTA described above.

Our results of aspirin-induced lipid peroxidation in liver are in agreement with the effect of the drug on stimulating MDA production by gastric mucosa (27) and erythrocytes (9). We are fully aware of the classical studies in which aspirin was shown to inhibit prostaglandin synthetase $(29,30)$, thereby decreasing arachidonic acid metabolism. Since MDA is a peroxidation product of arachidonic acid, an inhibitor of prostaglandin synthetase, such as aspirin, would be expected to inhibit production of MDA. Some authors assess prostaglandin synthetase activity by assaying for inhibition of MDA formation from arachidonic acid (31). Utilizing this assay, Roth et al. (31) have demonstrated that aspirin decreases prostaglandin synthetase (i.e., MDA formation from arachidonic acid) in sheep seminal vesicle microsomes. The type of effect on MDA production in response to aspirin is probably explained both by the varying iron content of the tissue studied as well as the dose of aspirin utilized (see Figs. 1 and 4). Both liver and erythrocytes contain large amounts of iron. When rats are given parenteral radioiron, the majority $(\sim 75 \%)$ is taken up by liver plus circulating red blood cells and marrow (32). Fur- 
thermore, ASA administered orally to rats increases the hepatic uptake of ${ }^{59} \mathrm{Fe}$ (33). In liver, apparently the effect of iron chelation by aspirin is predominant over the inhibition of prostaglandin synthetase by aspirin.

There are a number of possible sources of endogenous microsomal iron that might be available for chelation. Thomas and Aust (34) have recently shown that much of endogenous iron in microsomes is ferritin (83\% of the nonheme microsomal iron). However, note that Bonkowsky et al. (35) have demonstrated that the "microsomal iron content" depends on the technique utilized to isolate the microsomal fraction. In their studies, microsomes isolated by a calcium aggregation method ( 10 min centrifugation at $1,500 \mathrm{~g}$ ) contained less iron than those isolated by differential centrifugation (30 min centrifugation at $105,000 \mathrm{~g}$ ). This difference in iron levels may explain why MDA production in microsomes obtained by differential centrifugation in our studies and then incubated with $2.3 \mathrm{mM}$ aspirin was $44.6 \pm 1.7 \mathrm{nmol} \mathrm{MDA} / \mathrm{mg}$ protein compared with $27.2 \pm 1.8$ for values obtained using calcium aggregated microsomes incubated in the same concentration of aspirin.

At least in vitro, ferritin iron can be released by $\mathrm{O}_{\hat{2}}^{\bar{z}}$ with subsequent promotion of the peroxidation of microsomal lipids. However, it is not necessary to invoke ferritin as the site of iron release in liver microsomes. Montgomery et al. (36) as well as Thomas and Aust (34) have postulated the presence of a small, nonheme iron pool in microsomes, which is not ferritin, and, which is capable of promoting lipid peroxidation. Maines (37) has suggested that the iron released through the destruction of microsomal heme (mediated in her experiments by allylisopropylacetamide) promotes lipid peroxidation. Bonkowsky et al. (35) demonstrated that the iron content of rat liver mitochondria is approximately half that of microsomes. Our data, demonstrating that equivalent doses of aspirin promote less MDA formation in hepatic mitochondria than in microsomes (see Figs. 1 and 4), suggests that organelle iron content and MDA production correlate directly.

We suggest that our observations of in vitro aspirin-induced hepatic organelle lipid peroxidation may be of relevance to Reye's Syndrome, a hepatic encephalopathy of childhood in which a number of studies have demonstrated a strong epidemiologic association with aspirin intake (38-41). The link between our observations and Reye's Syndrome is all the more intriguing because of reports of products of lipid peroxidation in blood (42) and liver (43) of children with this syndrome. Since Bacon et al. (44) have demonstrated that in vivo iron loading in experimental animals is associated with evidence of lipid peroxidation in hepatic microsomes and mitochondria, our studies also raise the possibility of increased susceptibility to aspirin-induced hepatotoxicity in hemochromatosis. However, the medical literature does not contain any reports of such increased susceptibility.

Whereas caution must be exercised in extrapolating from our in vivo effects, it is of interest that Nakagawa et al. (45) have recently reported increased MDA in whole liver homogenates obtained from rats treated with several doses of oral aspirin. However, even this information does not necessarily establish a direct causal relationship between aspirin administration and tissue lipid peroxidation. As is the case with $\mathrm{CCl}_{4}$ and other hepatotoxicants, the administration of which is associated with evidence of hepatic lipid peroxidation, there is a gap in our understanding of the precise relationship between the initial reactant species, membrane lipid peroxidation, secondary mechanisms, such as changes in mitochondrial function and increased cytosolic calcium, and the eventual unfolding of histopathology (8).

In conclusion, our results suggest that ASA may chelate endogenous hepatic iron and thereby (either by direct or indirect mechanisms) promote lipid peroxidation. This hypothesis merits further investigation as a possible mechanism for aspirin-related hepatotoxicity.

\section{Acknowledgments}

We thank R. O. Recknagel, E. A. Glende, and W. P. Trumble for suggestions regarding experimental design, $S$. Dombek for expert secretarial assistance, and A. Kahn for critical review of the manuscript.

Supported in part by National Institutes of Health Bio-2 5 R23 NS20835-02 and by the John F. J. Mackiewicz Reye's Research Grant, St. Louis University Medical Center, Department of Pediatrics.

\section{References}

1. Schwarz, K. B., B. J. Arey, and S. Mahanty. 1986. Iron chelation by aspirin (ASA) as a possible mechanism for ASA-induced malondialdehyde (MDA) production in mouse liver microsomes. Hepatology (Baltimore). 6:1228. (Abstr.)

2. Schwarz, K. B., B. Arey, and S. Mahanty. 1987. Aspirin stimulates malondialdehyde production. J. Natl. Reye's Syndrome Foundation. 7:30-33.

3. Mahanty, S., B. J. Arey, and K. B. Schwarz. 1986. Aspirin stimulates malondialdehyde production in mouse liver microsomes. Pediatr. Res. 20:244. (Abstr.)

4. Zimmerman, H. J. 1981. Effects of aspirin and acetaminophen on the liver. Arch. Intern. Med. 141:333-342.

5. Kaplowitz, N., J. Kuhlenkamp, L. Goldstein, and J. Reeve. 1979. Effect of salicylates and phenobarbital on hepatic glutathione in the rat. J. Pharmacol. and Exp. Ther. 212:240-245.

6. Tolman, K., P. Peterson, P. Gray, and S. Hammar. 1978. Hepatoxicity of salicylates in monolayer cell cultures. Gastroenterology. 74:205-208.

7. Martens, M., and C. Lee. 1984. Reye's Syndrome: salicylates and mitochondrial functions. Biochem. Pharmacol. 33:2869-2876.

8. Recknagel, R. O., E. Glende, R. Waller, and K. Lourey. 1982. Lipid peroxidation: biochemistry, measurement and significance in liver cell injury. In Toxicology of the Liver. G. Plaa and W. Hewitt, editors. Raven Press, New York. 213-241.

9. Stockman, J., B. Lubin, and F. Oski. 1978. Aspirin-induced hemolysis: the role of concomitant oxidant $\left(\mathrm{H}_{2} \mathrm{O}_{2}\right)$ challenge. Pediatr. Res. 12:927-931.

10. Hunter, F. E. Jr., J. Gebicki, P. Hoffsten, J. Weinstein, and A. Scott. 1963. Swelling and lysis of rat liver mitochondria induced by ferrous ions. J. Biol. Chem. 238:828-835.

11. Hochstein, P., K. Nordenbrand, and I. Ernster. 1964. Evidence for the involvement of iron in the ADP-activated peroxidation of lipids in microsomes and mitochondria. Biochem. Biophys. Res. Commun. 14:323-328.

12. Wills, E. D. 1969. Lipid peroxide formation in microsomes. Biochem. J. 113:325-332.

13. Tien, M., L. A. Morehouse, J. R. Bucher, and S. D. Aust. 1982. The multiple effects of ethylenediaminetetraacetate in several model lipid peroxidation systems. Arch. Biochem. Biophys. 218:450-458.

14. Roders, M., E. Glende, and R. Recknagel. 1976. NADPH-dependent lipid peroxidation of calcium bound microsomes. Res. Commun. Chem. Pathol. Pharmacol. 15:393-396.

15. Recknagel, R., and A. Ghoshal. 1966. Lipoperoxidation as a vector in carbon tetrachloride hepatotoxicity. Lab. Invest. 15:132-148.

16. Lowry, O., N. Rosebrough, A. Farr, and R. Randall. 1951. 
Protein measurement with the Folin phenol reagent. J. Biol. Chem. 193:265-275.

17. Kornbrust, D. J., and R. D. Mavis. 1980. Microsomal lipid peroxidation. Mol. Pharmacol. 17:400-407.

18. Lowrey, K., E. A. Glende, and R. O. Recknagel. 1981. Destruction of liver microsomal calcium pump activity by carbon tetrachloride and bromotrichloromethane. Biochem. Pharmacol. 30:135-140.

19. Hogeboom, G. 1957. Fractionation of cell components of animal tissues. general preparative procedures. In Methods of Enzymology. S. Colowick and N. O. Kaplan, editors. Academic Press, New York. Vol. 3. 16-19.

20. Stookey, L. 1970. Ferrozine: a new spectrophotometric reagent for iron. Anal. Chem. 42:779-783.

21. Carter, P. 1971. Spectrophotometric determination of serum iron at the submigrogram level with a new reagent (ferrozine). Anal. Biochem. 40:450-458.

22. Williams, D., D. Walz, and W. Foye. 1976. Synthesis and biological evaluation of tetrakis (acetylsalicylato)-dicopper (II). $J$. Pharm. Sci. 65:126-128.

23. Ali, S. L. 1976. Application of gas-liquid chromatography and high-performance liquid chromatography to the analysis of trace amounts of salicylic acid, acetylsalicylic anhydride and acetylsalicylic acid in aspirin samples and aspirin formulations. J. Chromatogr. 126:651-663.

24. Rowland, M., S. Riegelman, P. A. Harris, S. D. Sholkoff, and E. J. Eyring. 1967. Kinetics of acetylsalicylic acid disposition in man. Nature (Lond.). 215:413-414.

25. Poyer, J. L., and P. B. McCay. 1971. Reduced triphosphopyridine nucleotide oxidase-catalyzed alterations of membrane phospholipids IV, Dependence of $\mathrm{Fe}^{3+}$. J. Biol. Chem. 246:263-269.

26. Sinaceur, J., C. Ribiere, J. Nordman, and R. Nordman. 1984. Superoxide degrading activity of desferrioxamine. In Oxygen Radicals in Chemistry and Biology. W. Bors, M. Saran, and D. Tait, editors. Walter de Gruyter, Berlin. 211-214.

27. Regillo, C., G. Pihan, and S. Szabo. 1984. Acetone potentiates and antioxidants diminish aspirin-induced lesions in gastric mucosa: possible role of lipid peroxidation. Gastroenterology. 86:1217. (Abstr.)

28. Martell, A., and M. Calvin. Methods of determining the presence of chelates.1952. In Chemistry of the Metal Chelate Compounds. Prentice-Hall Inc., Englewood Cliffs, NJ.

29. Vane, J. R. 1971. Inhibition of prostaglandin synthesis as a mechanism of action for aspirin-line drugs. Nat. New Biol. 231:232237.

30. Smith, J. B., and A. L. Willis. 1971. Aspirin selectively inhibits prostaglandin production in human platelets. Nat. New Biol. 231:235-237.
31. Roth, G. J., N. Stanford, and P. W. Majerus. 1975. Acetylation of prostaglandin synthase by aspirin. Proc. Natl. Acad. Sci. USA. 72:3073-3076.

32. Cheney, B. A., K. Lothe, E. H. Morgan, S. K. Soods, and C. A. Finch. 1967. Internal iron exchange in the rat. Am. J. Physiol. 212:376-380.

33. Glikman, P., A. Gutnisky, M. F. Gimeno, and A. L. Gimeno. 1980. Effect of acetylsalicylic acid on iron absorption in the rat. $E x$ perientia (Basel). 37:588-589.

34. Thomas, C. E., and S. D. Aust. 1985. Rat liver microsomal NADPH-dependent release of iron from ferritin and lipid peroxidation. J. Free Radicals. Biol. \& Med. 1:293-300.

35. Bonkowski, H. L., S. Carpenter, and J. Healey. 1979. Iron and the liver. Arch. Pathol. Lab. Med. 103:21-29.

36. Montgomery, M. R., C. Clark, and J. L. Holtzman. 1974. Iron species of hepatic microsomes from control and phenobarbital-treated rats. Arch. Biochem. Biophys. 160:113-118.

37. Maines, M. D. 1980 . Evidence for the catalytic activity of endogenous iron in the lipid peroxidative destruction of heme by allylisopropylacetamide in the rat liver. Int. J. Biochem. 12:781-785.

38. Starko, K. M., C. Ray, L. Dominquez, W. Stromberg, and D. F. Woodall. 1980. Reye's Syndrome and salicylate use. Pediatrics. 66:859-864.

39. Waldman, R. J., W. Hall, and H. McGee et al. 1982. Aspirin as a risk factor in Reye's Syndrome. JAMA (J. Am. Med. Assoc.). 247:3089-3094.

40. Halpin, T. J., F. Holtzhauer, and R. Campbell. 1982. Reye's Syndrome and medication use. JAMA (J. Am. Med. Assoc.). 248:687691.

41. Hurwitz, E. S., M. Barrett, and D. Bregman. 1987. Public health service study of Reye's Syndrome and medications. JAMA (J. Am. Med. Assoc.). 257:1905-1911.

42. Singh, Inderjit, Y. Yoshihiro, A. Singh, F. Tecklenberg, and C. Darby. 1987. Fatty acid metabolism in Reye's Syndrome. Pediatr. Res. 21:334. (Abstr.)

43. Brown, R. E., S. Koh, C. Bhuvaneswarran, and M. Brewster. 1982. Lipid peroxidation and hepatocellular degeneration in Reye's Syndrome. Ann. Clin. Lab. Sci. 12:327-328.

44. Bacon, B. R., A. Tavill, G. Brittenhan, C. Park, and R. Recknagel. 1983. Hepatic lipid peroxidation in vivo in rats with chronic iron overload. J. Clin. Invest. 71:429-439.

45. Nakagawa, M., N. Ishihara, T. Shimokawa, and S. Kojima. 1987. Effect of clofibrate on lipid peroxidation in rats treated with aspirin and 4-pentenoic acid. J. Biochem. (Tokyo). 101:81-88. 